\title{
An image analysis method for determining boiling front in minichannel heat exchanger
}

\author{
Gabriela Rafałko $^{1 *}$, Hubert Grzybowski ${ }^{1}$, Pawet Dzienis ${ }^{1}$, and Romuald Mosdorf ${ }^{1}$ \\ ${ }^{1}$ Department of Mechanics and Applied Computer Science, Faculty of Mechanical Engineering \\ Bialystok University of Technology, Wiejska 45 A, 15-351 Bialystok, Poland
}

\begin{abstract}
In this work a numerical method for determining boiling front in short time period of flow was presented. A non-stationary boiling in rectangular eleven minichannels $(0.25 \mathrm{~mm} \times 0.25 \mathrm{~mm} \times 32 \mathrm{~mm})$ was recorded using Phantom v1610 high speed camera with the speed of $6000 \mathrm{fps}$. In the algorithm correlation between subsequent frames was computed. Frames were divided into reference and test frames. In each iteration a part of a reference frame called 'reference gate' and moving part of a test frame called 'moving gate' were considered. A two-dimensional correlation coefficient was calculated. Such method allowed to identify the location of boiling front in each minichannel separately.
\end{abstract}

\section{Introduction}

A non-stationary flow boiling in minichannel is characterised by very rapid changes of phase distribution. The complexity of boiling phenomenon and the geometry of channels (e.g. small hydraulic diameter) result in occurrence of different instabilities (e.g. pressure drop, temperature, flow reversal, channel interaction). Thus, researchers still face difficulties to clearly describe this phenomenon [1]. Many approaches in the literature have been made which aim to study phase distribution in order to improve heat exchangers performance and analyse the boiling phenomenon [2]. The observations of boiling front were performed using, for instance, the liquid crystal thermography technique [3], analytical methods and simulations [4,5] and thermal imaging [6].

In the paper an image analysis method for determining moving boiling front position in minichannels is proposed.

\subsection{Experimental setup}

In the experimental setup shown in Fig. 1 distilled water was pumped by gear pump (Fig. 1 - 4) and flew through surge tank (Fig. 1 - 3). The water flow rate was measured by the mass flow meter (Fig. $1-5$ ). The exchanger was heated using electric power (Fig. $1-8$ ). The boiling temperatures were measured using seven thermocouples (Fig. 1 - 7). The inlet and outlet pressure was monitored by two pressure sensors (Fig. $1-6,9$ ). The heat exchanger (Fig. $1-1$ ) consisted of 11 parallel minichannels with the following dimensions: $0.25 \mathrm{~mm}$ x $0.25 \mathrm{~mm}$ (Fig. 1b), the channels length was $32 \mathrm{~mm}$, the wall between each channel was equal to $0.13 \mathrm{~mm}$. Two Translation Data Acquisition Systems (DAQ Data
Translation DT9804 and Data Translation DT9806) were used for recording all the signals at a sampling rate of $1 \mathrm{kHz}$.

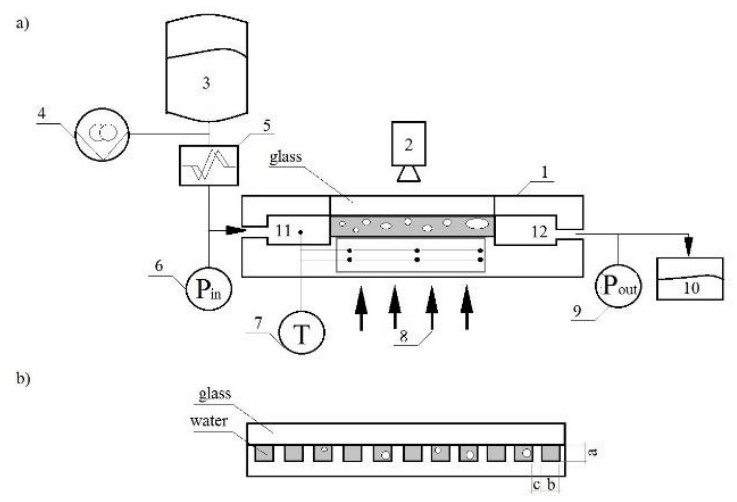

Fig. 1. Experimental setup: a) measurement path, $1-$ minichannel heat exchanger, 2 - high speed camera Phantom v1610,3 - surge tank, 4 - gear pump (Tuthill model DGS.11), 5 - mass flow meter (Bronkhorst mini CORIFLOW M13, accuracy of $\pm 0.2 \%$ of rate), 6 - inlet pressure sensor (MPX5050DP), 7 - a set of seven thermocouples (type $\mathrm{K}$, accuracy of $\pm 2.5^{\circ} \mathrm{C}$ ), 8 - heater, 9 - outlet pressure sensor (MPX5050DP), 10 - outlet tank, 11 - inlet of the heat exchanger, 12 - outlet of the heat exchanger, $b$ ) exchanger's cross-section: $\mathrm{a}=0.25 \mathrm{~mm}, \mathrm{~b}=0.25 \mathrm{~mm}, \mathrm{c}=0.13 \mathrm{~mm}$.

Flow boiling with different inlet water flow rates $\left(q_{w}\right)$ was considered (Tab. 1). The water flow rates $\left(q_{w}\right)$ and electric power of the heater $(P)$ for cases of flow boiling are presented in Table 1.

\footnotetext{
* Corresponding author: rafalkogabriela@gmail.com
} 
Table 1. The water flow rates $\left(q_{w}\right)$ and electric power of the heater $(P)$ for cases of flow boiling.

\begin{tabular}{|c|c|c|}
\hline Case no. & $\begin{array}{c}q_{w} \\
(\mathrm{~g} / \mathrm{h})\end{array}$ & $\begin{array}{c}P \\
(\mathrm{~W})\end{array}$ \\
\hline 1 & 180 & \multirow{2}{*}{5} \\
& \multirow{2}{*}{56} \\
\hline 2 & 255 & \\
\hline 3 & 370 & \\
\hline 4 & 435 & \\
\hline
\end{tabular}

Due to the fact that boiling in minichannels was a non-stationary process, the character of inlet pressure oscillations was changing (Fig. 2). The average value of the inlet pressure was changing in time. In order to consider this fact, four characteristic parts of the signal were extracted (when the average value of inlet pressure in the extracted part was equal to the maximum, minimum and average value of the registered signal). In such a way the considered parts of the signal had a different boiling intensity. When the average inlet pressure was high, then boiling was more intense in comparison with the case of lower inlet pressure. Four extracted parts of the signal for case no. 1 (Tab. 1) are presented in Fig. 2. The extracted video parts from each case of flow boiling lasted $0.5 \mathrm{~s}$ what corresponds to 3000 video frames. As a result, 16 different video parts of flow boiling were analysed.

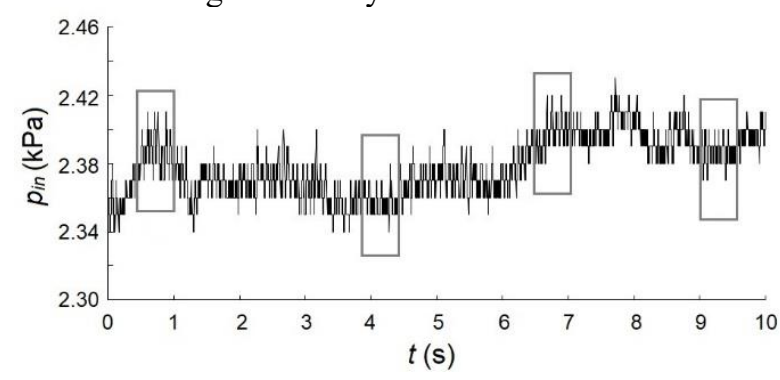

Fig. 2. The extracted parts from the registered signal of inlet pressure $\left(p_{i n}\right)$ for case no. 1 . The grey rectangles define the time periods in which the videos were analysed.

The example video frames for two cases of flow boiling are shown in Fig. 3. The analysed area of the frames was marked with a red square bracket (Fig. 3a).

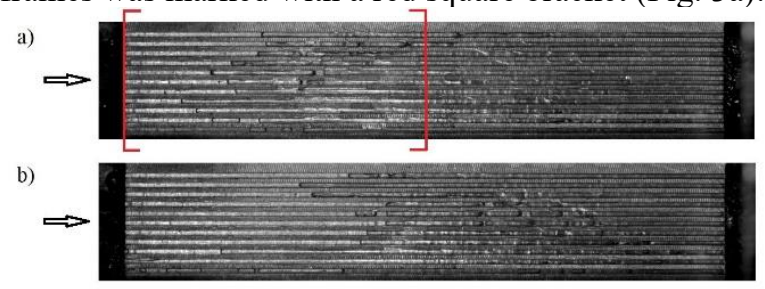

Fig. 3. An example video frame observed during: a) case no. 2 (Tab. 1), b) case no. 3 (Tab. 1); the flow direction was marked with an arrow; the analysed area of the frames was marked with a red square bracket.

\section{Analysis and modelling}

In the paper a method for determining the location of moving boiling front in a short time period during boiling was proposed. The algorithm is an extended version of the method presented in the paper [7].

In the proposed algorithm sets of video frames which consist of frames called 'reference frames' and 'test frames' are processed. The scheme of the algorithm is shown in Fig. 4. The reference frame (for $k=1$ ) and the test frame (for $j=1$ ) are shown in Fig. 4a. In each frame a part of the image is extracted which is called a 'gate'. The reference gate $G_{r}$ is marked with a red rectangle and the moving gate $G_{m}$ is marked with a blue rectangle (Fig. $4 \mathrm{a})$. The reference gate is denoted as $G_{r}\left(k, x_{r}\right)$ where $k$ indicates the number of the reference frame, $k \in\{1, \ldots, n$; $n \in N\}, x_{r}$ defines the distance of the gate from the channel inlet, $x_{r} \in\{1.25 \mathrm{~mm} * d, \ldots, f ; d \in N, f \in N\}$. The moving gate is denoted as $G_{m}\left(k+j^{*} \tau, x_{m}\right)$ where $x_{m}$ defines the position of the moving gate which is related to the reference gate's position: $x_{m}=x_{r}+i^{*} s$ where $i$ is the index of the gate's position, $i \in\{1, \ldots, g\}, s$ is the shift (in horizontal axis) of the gate related to the reference gate's position, $s \in\left\{0.05 \mathrm{~mm}^{*} h, \ldots, w ; h \in N, w \in N\right\}$ and $j$ is the index of the test frame, $j \in\{1,2, \ldots, t ; t \in N\}$. The time delay between subsequent test frames is denoted as $\tau$. A two-dimensional correlation coefficient defined as:

$$
R_{x m}\left(k, j, \tau, x_{r}\right)=\operatorname{cor}\left[\left(G_{r}\left(k, x_{r}\right), G_{m}\left(k+j^{*} \tau, x_{m}\right)\right]\right.
$$

is calculated between a reference gate $G_{r}$ in a position $x_{r}$ and moving gates $G_{m}$ situated on subsequent positions on a test frame $\left(x_{m}\right)$. The example of the $R_{x m}$ function for $\left(k=1, j=1, \tau=1, x_{r}=2.50 \mathrm{~mm}\right)$ is shown in Fig. 4b.

Next, for each analysed reference gate and its positions a function is obtained which is denoted as follows:

$$
S_{j}\left(k, x_{r}\right)=\max \left(R_{x m}\left(x_{r}\right)\right)-\operatorname{mean}\left(R_{x m}\left(x_{r}\right)\right)
$$

Two example $S_{j}$ functions for $k=1$ and $k=2$ are shown in Fig. 4c. In the next step the $S_{j}$ functions are averaged over $k$ reference gates. The averaged $S_{j}$ function is denoted as mean $\left(S_{j}\right)$. In the Fig. $4 \mathrm{~d}$ an example mean $\left(S_{j}\right)$ function averaged over $k=1$ and $k=$ 2 is shown. Two coefficients characterising the mean $\left(S_{j}\right)$ function are defined: its slope $(\alpha)$ and the first value $\left(\operatorname{mean}\left(S_{j}(1)\right)(\right.$ Fig. $4 \mathrm{~d})$. The slope $(\alpha)$ of the mean $\left(S_{j}\right)$ function calculated for several beginning points gives information about the changes of phase distribution in the channel while the first value, mean $\left(S_{j}(1)\right)$, presents the amplitude of changes which are observed in a short period of time (equal to $\tau$ ). In order to obtain a coefficient which is a measure of rate of phase distribution changes in minichannel the following function is defined:

$$
V_{v}\left(x_{r}\right)=-\alpha / \operatorname{mean}\left(S_{j}(1)\right)
$$

Finally, a position of the reference gate $x_{r}$, for which the $V_{v}$ coefficient reaches maximum (Fig. 4e) characterises the most probable position of the moving boiling front $\left(x_{b}\right)$ in the particular minichannel. This is because the maximum of $V_{v}$ indicates the location of the most rapid changes of phase distribution in the channel. The described procedure is performed for each minichannel of the heat exchanger. 
a)

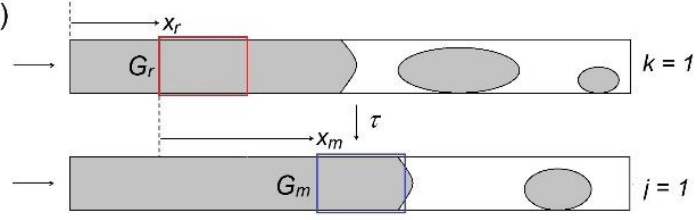

b)

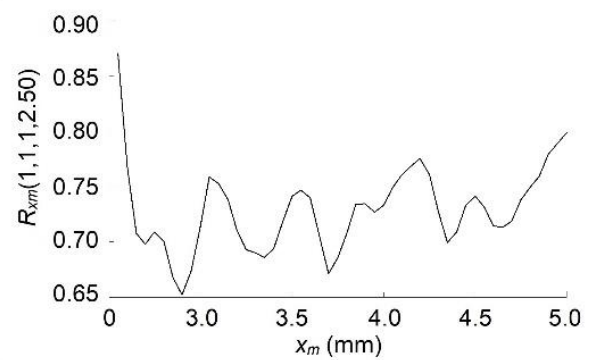

c)

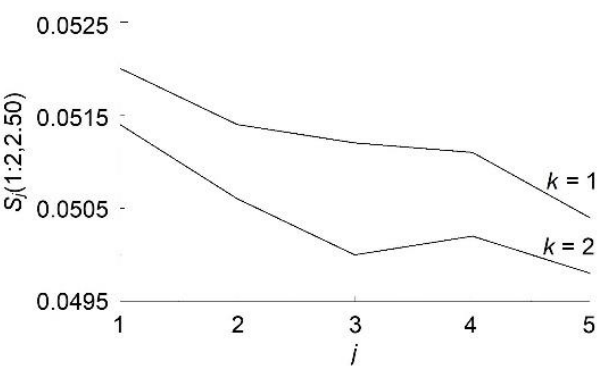

d)

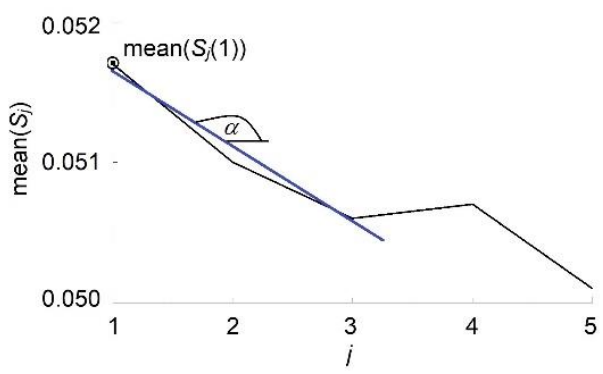

e)

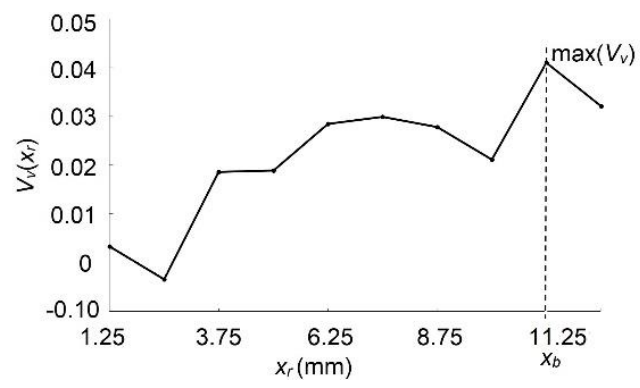

Fig. 4. Algorithm's steps: a) the reference gate $\left(G_{r}\right)$ and the moving gate $\left(G_{m}\right)$ and their relations, b) the correlation coefficient $\left(R_{x m}\right)$ vs. moving gate's positions $\left.\left(x_{m}\right), \mathrm{c}\right)$ the $S_{j}$ functions obtained for two example reference gates $(k=1, k$ $=2)$, d) the averaged $S_{j}$ function $\left(\operatorname{mean}\left(S_{j}\right)\right)$, e) the $V_{v}$ coefficient vs. the reference gate's position $\left(x_{r}\right), x_{b}-$ the position of boiling front, $\max \left(V_{v}\right)-$ maximum value of $V_{v}$.

In Fig. 5 a pseudocode of the proposed method is shown. The variable images includes video frames of flow boiling. In the pseudocode the dimensions of the images and time distance between susbsequent video frames correspond with the values defined in the next paragraph (in the pseudocode the dimensions of the frames are in pixels). The width and height of the gates are denoted respectively as: $w, h$. The vertical coordinates of the minihchannels are denoted as $y$. The variable $z$ is the number of the minichannel (counting from upper minichannel in the image - Fig. 3).

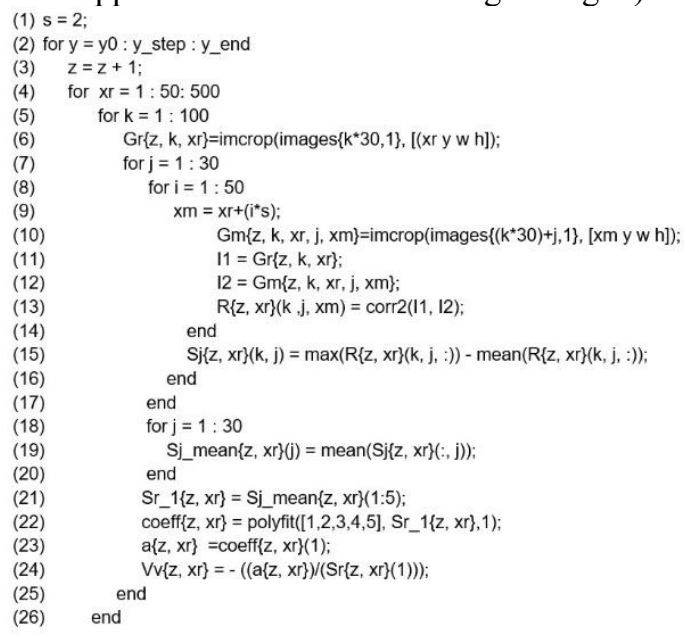

Fig. 5. The pseudocode of the proposed method implemented in Matlab R2014b software.

The analysis was performed for hundred subsequent reference frames $(k=100)$ delayed one from another by 30 frames $(0.005 \mathrm{~s})$ (Fig. 5 - lines 5,6). Next, thirty subsequent test frames $(j=30)$ which were situated right after each analysed reference frame were taken into consideration. The time delay between each test frame was $\tau=1(1.6 * 10-4 \mathrm{~s})$ (Fig. 5 - lines 7,10). The function 'imcrop' was used to extract the gates from video frames (Fig. $5-6,10$ ). The reference gate and moving gate dimensions were equal to: $0.10 \mathrm{~mm} \times 1.25 \mathrm{~mm}(4 \times 50$ pixels) (Fig. 5 - lines 6,10). The positions of the reference gates were following: $x_{r} \in\{1.25 \mathrm{~mm}, 2.50 \mathrm{~mm}$, ..., $12.50 \mathrm{~mm}$ \} moving from the inlet of the exchanger to the right (according to flow's direction) (Fig. 5 - lines 4,6). The shift of the moving gate counting from the beginning position of the refrence gate was equal to $s \epsilon$ $\{0.05 \mathrm{~mm}, 0.10 \mathrm{~mm}, \ldots, 2.50 \mathrm{~mm}\}$ moving respectively as in case of reference gates (Fig. 5 - lines 1,9,10). The functions defined in the algorithm are defined in lines 13:24 of the pseudocode shown in Fig. 5. The slope of mean $\left(S_{j}\right)$ function was calculated using the function 'polyfit' for five beginning points (Fig. 5 - lines $21,22,23)$. The algorithm was used to calculate the boiling front location in each of ten minichannels (due to the poor lighting in case of the $11^{\text {th }}$ minichannel) (Fig. 5 - line 2).

\section{Results and discussion}

Figure 6 presents the positions of boiling front $\left(x_{b}\right)$ averaged over 10 minichannels vs. the water flow rate $\left(q_{w}\right)$. Additionaly, Fig. 6 shows the result of a rough estimation of the Hurst exponent $(H)$ of the position changes of the boiling front in each of ten minichannels:

$$
H=\log (R / S) / \log (N)
$$

where $N$ - the length of data set $(N=10), R$ - the range of the values of the boiling front position changes in all considered minichannels, $S$ - the standard deviation of 
the position changes of the boiling front in all considered minichannels.

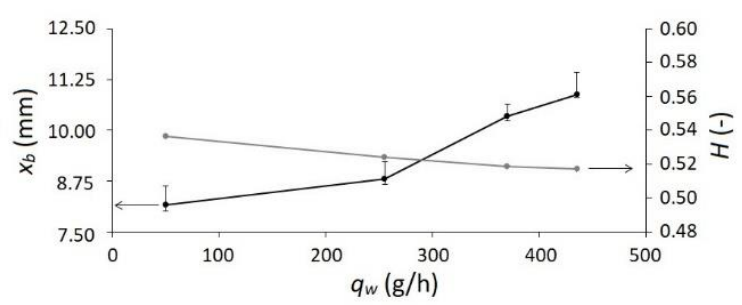

Fig. 6. The boiling front locations $\left(x_{b}\right)$ and Hurst exponents $(H)$ vs. water flow rate $\left(q_{w}\right)$ for flow boiling cases.

The lowest value of Hurst exponent was obtained for the highest water flow rate. This indicates that changes of boiling front position in subsequent minichannels occured more chaotically than in case of higher values of Hurst exponents (Fig. 6).

Figure 7 present positions of boiling front (marked with dots) and their standard deviation obtained for case no. 2 (black) and 3 (grey).

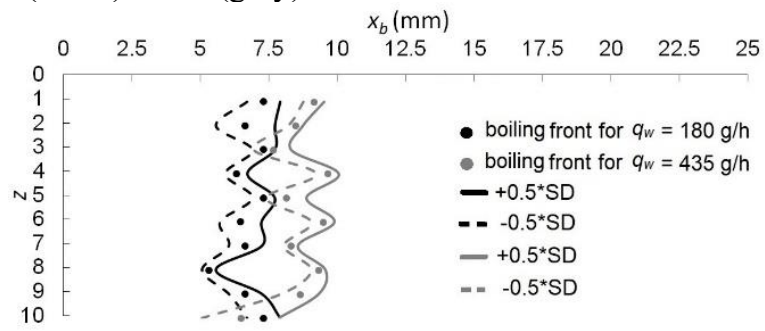

Fig. 7. The boiling front locations $\left(x_{b}\right)$ and standard deviation (SD) calculated for: case no. 1 (marked with black), case no. 4 (marked with grey).

\section{Conclusions}

In order to assess the accuracy of the method the following procedure was applied. The subsequent steps of evaluation procedure are presented based on the data from case no. 1 , part 1 which is presented in Tab. 2 and Tab. 3. In Tab. 2 column $z$ denotes the minichannel number. The next six columns contain boiling front locations in each minichannel obtained for 6 different video parts. Two last columns include average value (avr) and standard deviation $(S D)$ of the positions of boiling front calculated for 6 videos, separately for each minichannel. Table 2 was prepared for all the cases and all the analysed video parts. In total in our analysis were 16 such tables. Next, based on those 16 tables the position of the boiling front was averaged over all minichannels. This way a representative boiling front position for each case was obtained. The result is shown in the second column of Tab. 3. Table 3 also presents an estimated error $(\varepsilon)$ of boiling front position defined as the percentage of quotient of standard deviation and average front position calculated for each minichannel. In each averaging operation of the boiling front position minimum and maximum values of the error were determined. This way, minimum and maximum values of the error for each case shown in Tab. 3 were assessed.

Table 2. The example calculations for case no. 1, part 1 performed during the evaluation of the method; $S D$ standard deviation of boiling front positions for 6 different videos, $a v r$ - average value of boiling front positions for 6 different videos.

\begin{tabular}{|c|c|c|c|c|c|c|}
\hline \multicolumn{7}{|c|}{ Case no. 1 , Part 1} \\
\hline \multicolumn{7}{|c|}{ video numbers } \\
\hline$z$ & 1 & 2 & $\ldots$ & 6 & $a v r$ & $S D$ \\
\hline$(-)$ & \multicolumn{4}{|c|}{$x_{b}(\mathrm{~mm})$} & \multicolumn{2}{|c|}{$(\mathrm{mm})$} \\
\hline 1 & 10.00 & 11.25 & \multirow[t]{10}{*}{$\ldots$} & 7.50 & 9.58 & 1.91 \\
\hline 2 & 8.75 & 12.50 & & 7.50 & 9.58 & 2.60 \\
\hline 3 & 8.75 & 8.75 & & 11.25 & 9.58 & 1.44 \\
\hline 4 & 8.75 & 6.25 & & 8.75 & 7.92 & 1.44 \\
\hline 5 & 10.00 & 7.50 & & 8.75 & 8.75 & 1.25 \\
\hline 6 & 7.50 & 10.00 & & 8.75 & 8.75 & 1.25 \\
\hline 7 & 10.00 & 10.00 & & 7.50 & 9.17 & 1.44 \\
\hline 8 & 7.50 & 6.25 & & 6.25 & 6.67 & 0.72 \\
\hline 9 & 6.25 & 8.75 & & 10.00 & 8.33 & 1.91 \\
\hline 10 & 10.00 & 7.50 & & 11.25 & 9.58 & 1.91 \\
\hline
\end{tabular}

Table 3. The results of the evaluation procedure; $a v r-$ boiling front positions averaged over all minichannels and all parts of the signal; minimum and maximum values of the error $(\varepsilon)$ determined for all minichannels and all parts of the signal.

\begin{tabular}{|c|c|c|c|}
\hline Case no. & $a v r$ & $\min (\varepsilon)$ & $\max (\varepsilon)$ \\
\hline$(-)$ & $(\mathrm{mm})$ & $(\%)$ & $(\%)$ \\
\hline 1 & 8.16 & 6 & 37 \\
\hline 2 & 8.83 & 6 & 35 \\
\hline 3 & 10.35 & 0 & 24 \\
\hline 4 & 10.88 & 0 & 43 \\
\hline
\end{tabular}

The boiling phenomenon in minichannel has a very rapid character, thus the flow boiling videos were registered with high speed. In order to assess a representative boiling front position for each flow, the described evaluation procedure included several averaging operations. In such a way, the boiling front positions determined for each case included the information about the phase distribution changes occurring in individual minichannels. The maximum error $(\varepsilon)$ of boiling front location was equal to $43 \%$ which is about $4.68 \mathrm{~mm}(\sim 15 \%$ of the channel length). In two out of four analysed cases the minimum error was close to $0 \%$ which means that the determined location of the boiling front for different parts of the signal was the same in each iteration of the evaluation procedure. One can notice that the boiling front positions determined in the paper are in a form of an area. This area represents the most rapid phase distribution changes and is equal to the dimensions of the reference gate $0.10 \mathrm{~mm} \times 1.25 \mathrm{~mm}$ ( 4 x 50 pixels). The reference gate covers the middle part of the width of the minichannel ( $40 \%)$ and about $4 \%$ of the length of the minichannel.

The results indicate that the proposed image analysis method is a non-invasive, efficient tool in boiling phenomenon studies. The paper also reveals that the boiling front is not only a point or a curve but a specific area of the heat exchanger.

This work was supported by the National Science Centre, Poland [Grant Number: UMO-2017/27/B/ST8/02905].

Project financing through the program of the Minister of Science and Higher Education of Poland named "Regional 
Initiative of Excellence" in 2019 - 2022 project number $011 /$ RID / 2018/19 amount of financing 12,000,000 PLN.

\section{References}

1. Y. K. Prajapati, P. Bhandari, Exp. Therm. Fluid Sci. 88, 576-593 (2017)

2. M. Sardeshpande, V. Ranade, Sadhana 38(6), 1083-1126 (2013)

3. M. Piasecka, Metrol. Meas. Syst. 20(2), 205-216 (2013)

4. K. Pruess, An analytical solution for heat transfer at a moving boiling front, Lawrence Berkley National Laboratory, LBNL Report \#: LBL-22513 (1987)

5. F. Krause, S. Schüttenberg, U. Fritsching, Int. J. Numer. Method. H. 20(3), 312-331 (2010)

6. T. -L. Liu, C. Pan, App. Therm. Eng. 94, 568-578 (2016)

7. G. Rafałko, R. Mosdorf, G. Górski, Int. Commun. Heat Mass Transf., 113, 104508 (2020) 